\title{
Acute viral bronchiolitis in South Africa: Strategies for management and prevention
}

\author{
H J Zar, ${ }^{1}$ MB BCh, FC Paed (SA), PhD; S A Madhi, ${ }^{2}$ MB BCh, MMed (Paed), FC Paed (SA), PhD; \\ D A White, ${ }^{3} \mathrm{MB}$ BCh, FC Paed (SA), MMed (Paed), Dip Allerg (SA), Cert Pulmonology (SA) Paed; \\ R Masekela, ${ }^{4}$ MB BCh, MMed (Paed), Cert Pulmonology (SA) Paed, Dip Allerg (SA), FCCP, PhD; \\ S Risenga, ${ }^{5}$ MB ChB, FC Paed (SA), Dip Allerg (SA), Cert Pulmonology (SA) Paed; H Lewis, ${ }^{6}$ MB ChB, MMed; \\ C Feldman, ${ }^{7}$ MB BCh, FCP (SA), FRCP, PhD, DSc; B Morrow, ${ }^{8}$ BSc (Physio), PG Dipl (Health Research Ethics), PhD; \\ P Jeena, ${ }^{4}$ MB ChB, FC Paed (SA), Cert Pulmonology (SA) Paed
}

\begin{abstract}
${ }^{1}$ Department of Paediatrics and Child Health, Red Cross War Memorial Children's Hospital, and MRC Unit on Child and Adolescent Health, Faculty of Health Sciences, University of Cape Town, South Africa

${ }^{2}$ Medical Research Council: Respiratory and Meningeal Pathogens Research Unit, University of the Witwatersrand, Johannesburg, South Africa

${ }^{3}$ Department of Paediatrics and Child Health, Faculty of Health Sciences, University of the Witwatersrand, Johannesburg, South Africa

${ }^{4}$ Department of Paediatrics and Child Health, Faculty of Health Sciences, University of KwaZulu-Natal, Durban, South Africa

${ }^{5}$ Department of Pulmonology, Faculty of Health Sciences, University of Limpopo, Polokwane, and Pietersburg Hospital, South Africa

${ }^{6}$ Department of Paediatrics and Child Health, Faculty of Health Sciences, University of Pretoria, South Africa

${ }^{7}$ Department of Medicine, Faculty of Health Sciences, University of the Witwatersrand, Johannesburg, South Africa

${ }^{8}$ Department of Paediatrics and Child Health, Faculty of Health Sciences, University of Cape Town, South Africa
\end{abstract}

Corresponding author: H Zar (heather.zar@uct.ac.za)

\begin{abstract}
Management of acute viral bronchiolitis is largely supportive. There is currently no proven effective therapy other than oxygen for hypoxic children. The evidence indicates that there is no routine benefit from inhaled, rapid short-acting bronchodilators, adrenaline or ipratropium bromide for children with acute viral bronchiolitis. Likewise, there is no demonstrated benefit from routine use of inhaled or oral corticosteroids, inhaled hypertonic saline nebulisation, montelukast or antibiotics. The last should be reserved for children with severe disease, when bacterial co-infection is suspected.

Prevention of respiratory syncytial virus (RSV) disease remains a challenge. A specific RSV monoclonal antibody, palivizumab, administered as an intramuscular injection, is available for children at risk of severe bronchiolitis, including premature infants, young children with chronic lung disease, immunodeficiency, or haemodynamically significant congenital heart disease. Prophylaxis should be commenced at the start of the RSV season and given monthly during the season. The development of an RSV vaccine may offer a more effective alternative to prevent disease, for which the results of clinical trials are awaited.

Education of parents or caregivers and healthcare workers about diagnostic and management strategies should include the following: bronchiolitis is caused by a virus; it is seasonal; it may start as an upper respiratory tract infection with low-grade fever; symptoms are cough and wheeze, often with fast breathing; antibiotics are generally not needed; and the condition is usually self limiting, although symptoms may occur for up to 4 weeks in some children.
\end{abstract}

S Afr Med J 2016;106(4):330-332. DOI:10.7196/SAMJ.2016.v106i4.10437

\section{Management of bronchiolitis}

Management of acute bronchiolitis is largely supportive. ${ }^{[1]}$ There is currently no proven effective therapy other than oxygen for hypoxic children (Table 1). . $^{[2,3]}$

The following supportive treatments have been used for the management of bronchiolitis:

- humidified oxygen

- inhaled short-acting bronchodilator therapy

- nebulised hypertonic saline (3\% or $5 \%$ )

- corticosteroids: oral or nebulised

- inhospital use of antiviral treatments, e.g. ribavirin in ventilated children

- montelukast

- antibiotics.

Humidified low-flow oxygen $(0.5-3.0 \mathrm{~L} / \mathrm{min})$ applied by nasal prongs is effective for hypoxic children. Nasal prongs give a maximum inspired oxygen of $28-35 \%$ except in small infants, when higher oxygen concentrations may be obtained. Headbox oxygen is an alternative and is well tolerated by young

Table 1. Therapies that may be beneficial in the management of bronchiolitis and those that are currently not routinely indicated

Beneficial therapies
Humidified oxygen
Therapies not routinely indicated
Bronchodilators
Adrenaline
Steroids (oral, systemic or nebulised)
Montelukast
Physiotherapy
Mucolytics and decongestants

infants. It requires no humidification, but high flow and a mixing device are needed to ensure that the correct oxygen concentration is delivered. However, there is wastage of oxygen and the delivered oxygen concentration $\left(\mathrm{FiO}_{2}\right)$ is unpredictable. Facemask oxygen delivers between $28 \%$ and $65 \%$ oxygen at a flow rate of $6-10 \mathrm{~L} / \mathrm{min}$. In severely hypoxic infants who are not ventilated, oxygen should be administered using a polymask, which enables $\mathrm{FiO}_{2}$ concentrations of $60-80 \%$ being achieved. Oxygen should be weaned when the child improves clinically and as hypoxia resolves.

Rapid, short-acting inhaled bronchodilator therapy such as albuterol or salbutamol has not shown any important clinical benefits in the treatment of bronchiolitis. ${ }^{[1,4]}$ A Cochrane review (30 trials, 1992 infants, including inpatient and emergency settings) reported no effects 
in oxygen saturation, reduction in hospital admission after outpatient treatment, duration of hospitalisation or time to resolution of illness at home. ${ }^{[4]}$ Given the potential for adverse side-effects and the cost of these treatments, bronchodilators should not be recommended for the routine management of bronchiolitis. For studies of adrenaline compared with placebo, a Cochrane review (19 studies, 2256 children) suggested a short-term benefit from adrenaline, especially in the first 24 hours of the illness. ${ }^{[5]}$ No differences were found for length of hospital stay, but there was some evidence that adrenaline was effective for reducing the number of hospital admissions. ${ }^{[5]}$ One large, high-quality trial suggested that combined treatment with dexamethasone and adrenaline may significantly reduce the number of admissions. ${ }^{[5]}$ However, there is currently insufficient evidence to support the use of adrenaline for the treatment of bronchiolitis among children admitted to hospital. Inhaled ipratropium bromide is an ineffective treatment. ${ }^{[6]}$

Conflicting data have been reported for the efficacy of hypertonic saline nebulisation administered as nebulised $3 \%$ or $5 \%$ saline for acute bronchiolitis. A 2013 Cochrane review (11 trials, 1090 children) reported a reduction in duration of hospital stay and improvement in clinical scores in children who were inpatients, but no short-term effects in children in four trials done in an emergency unit setting. ${ }^{[7]}$ However, recently the largest reported randomised controlled study of nebulised hypertonic saline in acute bronchiolitis: randomised controlled trial and economic evaluation (SABRE) in hypoxic children, found no difference in outcomes between children who received hypertonic saline compared with those who received standard care. ${ }^{[8]}$ Other recently published randomised trials have also added to the evidence against the use of hypertonic saline in bronchiolitis, showing no difference in length of hospital stay, clinical scores or improvement in oxygenation compared with children receiving normal saline nebulisation or salbutamol. ${ }^{[9-12]}$ The current evidence does not demonstrate consistent benefit with the use of hypertonic saline; currently, this strategy should therefore not be recommended.

Systemic or inhaled corticosteroids have been shown not to be effective in reducing hospital admission or improving clinical scores in ambulatory patients. ${ }^{[1,13]}$ However, among inpatients, corticosteroids improved clinical scores within the first 12 hours, but did not have any effect on length of stay. Therefore, corticosteroids should not be routinely recommended. ${ }^{[13]}$

Five randomised controlled trials have shown no evidence of benefit for inhaled corticosteroids started in the acute phase of bronchiolitis for prevention of post-bronchiolitic wheezing. ${ }^{[14]}$ Routine use of systemic or inhaled steroids in the management of bronchiolitis is therefore not indicated.

Montelukast has no effect on the clinical course or outcome of bronchiolitis. A study of montelukast ( $4 \mathrm{mg}$ daily until discharge) found that it demonstrated no improvement in the clinical course of the disease. ${ }^{[15]}$ In a study of post-bronchiolitis wheeze, montelukast did not improve respiratory symptoms of post-RSV bronchiolitis in children. ${ }^{[16]}$

Similarly, aerosolised ribavirin has been reported not to have any significant consistent beneficial effect in the management of bron-

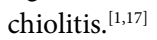

The use of chest physiotherapy has been shown not to change the course of bronchiolitis or its outcome. Chest physiotherapy using vibration and percussion techniques does not reduce the length of hospital stay or oxygen requirements or improve the severity clinical scores in infants with acute bronchiolitis. ${ }^{[18]}$

Finally, a Cochrane review of antibiotics compared with placebo for bronchiolitis, including two studies of azithromycin compared with placebo, found no difference in length of hospital stay, duration of oxygen administration or readmission rates. ${ }^{[19]}$ Antibiotics should therefore not be used routinely in bronchiolitis, except in children with severe disease in whom bacterial lower respiratory tract infection is suspected.

\section{Prevention of $\mathbb{R S V}$ disease in high-risk chilldren.}

The specific RSV monoclonal antibody, palivizumab, is available for children at risk of severe bronchiolitis.

With regard to RSV-associated morbidity, the risk of hospitalisation is $5.2 / 1000\left(4.8\right.$ - 5.7) cases. $^{[20]}$ However, this risk rapidly increases with decreasing gestational age, being 19.3/1 000 in premature infants $<29$ weeks of gestational age and with congenital heart defects. RSV hospitalisation rates are highest at 120.8/1 000 in the first 6 months after delivery, declining to $63.2 / 1000$ at $6-12$ months of age and to $18.2 / 1000$ in the $>12$-month age group. ${ }^{[20]}$ Similarly, the risk of hospitalisation in children with chronic lung disease of prematurity is 562.5/1 000 in the $<6$-month age group, 214.3/1 000 in the 6 - 12-month age group, and 73.4/1 000 in those $>12$ months of age. ${ }^{[20]}$

Palivizumab has been demonstrated to be effective in reducing RSV-related hospitalisation and the need for intensive care unit (ICU) admission among all premature infants and those with bronchopulmonary dysplasia by $55 \%$, with an adjusted risk reduction of 3 - 9\% in a Level 1 study. ${ }^{[21]}$ Meta-analysis has confirmed this across all populations of preterm infants. ${ }^{[22]}$ This monoclonal antibody has also been shown to reduce duration of hospitalisation stay and need for oxygen in young children with congenital heart defects. ${ }^{[23]}$ Given the current burden of RSV disease, it is estimated that the number needed to treat to prevent one hospitalisation from RSV disease in premature infants and children with chronic lung disease or congenital heart defects is between 16 and 23, which increases sixfold for the prevention of ICU admission or death. At the current cost of palivizumab, it is extremely expensive to recommend its widespread use; it is therefore restricted to high-risk groups. ${ }^{[24]}$

For the prevention of RSV-associated bronchiolitis, the South African (SA) guideline for the use of palivizumab recommends that it should be restricted for use in the first 6 months of life in highrisk children, defined as premature infants. ${ }^{[21,22]}$ Furthermore, infants with chronic lung disease of prematurity or those with congenital heart defects with significant haemodynamic instability (complex lesions with pulmonary hypertension) and the premature neonate who is a graduate of an ICU and has ongoing respiratory or cardiac compromise (diuretic, oxygen or corticosteroid dependent), should be covered during the first 24 months of life and during the RSV season. RSV prophylaxis may be considered in children with profound immunocompromise or pulmonary neuromuscular disease. The value of palivizumab is uncertain in children with Down syndrome, cystic fibrosis, recurrent wheeze and in nosocomial outbreaks.

It would be appropriate to use palivizumab between January and May in high-risk infants ( $<6$ months old), as the RSV season in SA is between February and June. ${ }^{[25]}$ The standard dose of $15 \mathrm{mg} / \mathrm{kg}$ given monthly for 5 months is advocated. All risk groups who are resident in hospital and for whom palivizumab is indicated, should be initiated on palivizumab in hospital as per guidelines, and not only after they are discharged.

\section{Indications for palivizumab}

Indications for palivizumab are the following:

- Premature infants of gestational age of $<36$ weeks at birth and $<6$ months of age at the start of the RSV season (February). Prophylaxis should be continued until the end of the RSV season (last dose in May). 
- Children of any gestation and $<24$ months of age at the start of the RSV season, with any of the following: chronic lung disease of prematurity, chronic lung disease, primary immunodeficiency, haemodynamically significant congenital heart disease.

- High-risk premature infants should commence their prophylaxis while still in hospital.

\section{RSV vaccine}

While RSV vaccine development was stalled for many years owing to the adverse effects associated with a formalin-inactivated vaccine, substantial progress has recently been made in the development of a possible vaccine. ${ }^{[26]}$ There are several candidate vaccines undergoing Phase 1 trials and two undergoing Phase 2 studies (a live attenuated vaccine for immunisation of infants and an F-protein vaccine for immunisation of pregnant women as a strategy to protect infants). A Phase 3 study, using a novel strategy of immunisation of pregnant women in their last trimester of pregnancy, has begun. This strategy will potentially be effective to prevent disease in young infants by transplacental antibody transfer and prevention of infection in mothers. Such a strategy may have to be coupled with vaccination of infants to enable more protection of young children for the first two years of life.

\section{Parent and caregiver education}

Management of children with bronchiolitis requires that parents or caregivers be educated about the condition. This is critical when children are not admitted to hospital, but also after discharge. The key elements of an educational message are listed in Table 2.
Table 2. Key elements of an educational message for parents of children with bronchiolitis

The condition may start as an upper respiratory tract infection with low-grade fever

Symptoms are cough and wheeze and often fast breathing Bronchiolitis is caused by a virus; antibiotics are not needed Bronchiolitis is usually self limiting, although symptoms may occur for up to 4 weeks in some children

\section{References}

1. Castro-Rodriguez JA, Rodriguez-Martinez CE, SossaBriceño MP. Principal findings of systematic reviews for the Briceno MP. Principal findings of systematic reviews for the
management of acute bronchiolitis in children. Paediatr Respir Rev 2015;15:S1526-1542.

2. Rojas-Reyes MX, Granados Rugeles C, Charry-Anzola LP. Oxygen therapy for lower respiratory tract infections in children between 3 months and 15 years of age. Cochrane Database Syst Rev 2014;12:CD005975. DOI:10.1002/14651858.CD005975.pub3

3. Beggs S, Wong ZH, Kaul S, Ogden KJ, Walters JA. High-flow nasal cannula therapy for infants with bronchiolitis. Cochrane Database Syst Rev 2014;1:CD009609. DOI:10.1002/14651858. CD009609.pub2

4. Gadomski AM, Scribani MB. Bronchodilators for bronchiolitis. Cochrane Database Syst Rev 2014;6:CD001266. DOI 10.1002/14651858.CD001266.pub4.CD001266

5. Hartling L, Bialy LM, Vandermeer B, et al. Epinephrine for bronchiolitis. Cochrane Database Syst Rev 2011;6:CD003123. bronchiolitis. Cochrane Database Syst Rev 2 10.1002/14651858.CD003123.pub3
DOI:

6. Henry RL, Milner AD, Stokes GM. Ineffectiveness of ipratropium 6. Henry RL, Milner AD, Stokes GM. Ineffectiveness of ipratropium
bromide in acute bronchiolitis. Arch Dis Child 1983;58:925-926. DOI:10.1136/adc.58.11.925

7. Zhang L, Mendoza-Sassi RA, Wainwright C, Klassen TP Nebulised hypertonic saline solution for acute bronchiolitis in infants. Cochrane Database Syst Rev 2013;7:CD006458. DOI 10.1002/14651858.CD006458.pub3

8. Everard ML, Hind D, Ugonna K, et al. SABRE: A multicentre randomised control trial of nebulised hypertonic saline in infants hospitalised with acute bronchiolitis. Thorax 2014;69:1105-1112. DOI:10.1136/thoraxjnl-2014-205953

9. Sharma BS, Gupta MK, Rafik SP. Hypertonic (3\%) saline vs $0.93 \%$ saline nebulization for acute viral bronchiolitis: A randomized controlled trial. Indian J Pediatr 2013;50:743-477. DOI:10.1007/s13312-013-0216-8

10. Teunissen J, Hochs AH, Vaessen-Verberne A, et al. The effect of $3 \%$ and $6 \%$ hypertonic saline in viral bronchiolitis: A randomised controlled trial. Eur Respir J 2014;44:913-921. DOI $10.1183 / 09031936.0015961$

11. Wu S, Baker C, Lang ME, et al. Nebulized hypertonic saline for bronchiolitis: A randomized clinical trial. JAMA Pediatr 2014;168:657-663. DOI:10.1001/jamapediatrics.2014.301
12. Jacobs JD, Foster M, Wan J, et al. 7\% hypertonic saline in acute bronchiolitis: A randomized controlled trial. Pediatrics 2014;133:e8-e13. DOI:10.1542/peds.2013-1646

13. Fernandes RM, Bialy LM, Vandermeer B, et al. Glucocorticoids for acute viral bronchiolitis in infants and young children for acute viral bronchiolitis in infants and young children,
Cochrane Database Syst Rev 2013;6:CD004878. DOI Cochrane Database Syst Rev
10.1002/14651858.CD004878.pub4

14. Blom D, Ermers M, Bont L, van Aalderen WM, van Woensel JB. Inhaled corticosteroids during acute bronchiolitis in the prevention of post-bronchiolitic wheezing. Cochrane Database Syst Rev 2007;1:CD004881. DOI:10.1002/14651858. CD004881.pub3

15. Amirav I, Luder AS, Kruger N, et al. A double-blind, placebocontrolled, randomized trial of montelukast for acute bronchiolitis. controlled, randomized trial of montelukast for acute bronchio

16. Peng WS, Chen X, Yang XY, Liu EM. Systematic review of montelukast's efficacy for preventing post-bronchioliti wheezing. Pediatr Allergy Immunol 2014;25(2):143-150. DOI 10.1111/pai.12124

17. Ventre K, Randolph A. Ribavirin for respiratory syncytial virus infection of the lower respiratory tract in infants and young children. Cochrane Database Syst Rev 2007;1:CD000181. DOI $10.1002 / 14651858$

18. Roqué i Figuls M, Giné-Garriga M, Granados Rugeles C, Perrotta C. 0 and 24 months old. Cochrane Database Syst Rev 2012;2:CD004873. DOI: 10.1002/14651858.CD004873.pub4

19. Farley R, Spurling GK, Eriksson L, Del Mar CB. Antibiotics for bronchiolitis in children under two years of age. Cochrane for bronchiolitis in children under two years of age. Cochrane
Database Syst Rev 2014;10:CD005189. DOI:10.1002/14651858. Database Syst
cd005189.pub4

20. American Academy of Pediatrics Committee on Infectious Diseases; American Academy of Pediatrics Bronchiolitis Guidelines Committee. Updated guidance for palivizumab prophylaxis among infants and young children at increased risk of hospitalization for respiratory syncytial viru infection. Pediatrics 2014;134(2):415-420. DOI:10.1542/ peds.2014-1665

21. The Impact-RSV study Group. Palivizumab, a humanized respiratory syncyctial virus monoclonal antibody, reduces hospitalization from respiratory syncytial virus infection in high risk infants. Pediatrics 1998;102:531-537. DOI:10.1542/ peds.102.3.531

22. Wegzyn C, Toh LK, Notario G, et al. Safety and effectiveness of palivizumab in children at high risk of serious disease due to respiratory syncytial virus infection: A systematic review. Infect respiratory syncytial virus
Dis Ther 2014:3:133-158.

23. Feltes TF, Cabalka AK, Meissner HC, et al., Cardiac Synagis study group. Palivizumab prophylaxis reduces hospitalization due to respiratory syncytial virus in young children with haemodynamically significant congenital heart disease. J Pediat 2003;143:532-540. DOI:10.1067/S0022-3476(03)00454-2

24. Wang D, Bayliss S, Meads C. Palivizumab for immunoprophylaxis of respiratory syncytial virus (RSV) bronchiolitis in high risk infants and young children: A systematic review and additional economical modelling of subgroup analyses. Health Technol Assess 2011;15:9971018. DOI: $10.3310 /$ hta 15050

25. Pretorius MA, Madhi SA, Cohen C, et al. Respiratory viral coinfections identified by a 10-plex real-time reverse-transcription polymerase chain reaction assay in patients hospitalized with polymerase chain reaction assay in patients hospitalized with
severe acute respiratory illness - South Africa, $2009-2010$. Infect Dis 2012;206(S1):S159-S65. DOI:10.1093/infdis/jis538

26. Resch B. Respiratory syncytial virus infection in high-risk infants - an update on palivizumab prophylaxis. Open Microbiol J 2014;8:71-77. DOI:10.2174/187428580140801007 